\title{
Article \\ On the Emergence of Orientational Order in Folded Proteins with Implications for Allostery
}

\author{
Debayan Chakraborty (D), Mauro Lorenzo Mugnai (D) and D. Thirumalai * (D) \\ Department of Chemistry, The University of Texas at Austin, Austin, TX 78712, USA; \\ debayan.chakraborty@utexas.edu (D.C.); mmugnai@utexas.edu (M.L.M.) \\ * Correspondence: dave.thirumalai@gmail.com
}

\begin{abstract}
The beautiful structures of single- and multi-domain proteins are clearly ordered in some fashion but cannot be readily classified using group theory methods that are successfully used to describe periodic crystals. For this reason, protein structures are considered to be aperiodic, and may have evolved this way for functional purposes, especially in instances that require a combination of softness and rigidity within the same molecule. By analyzing the solved protein structures, we show that orientational symmetry is broken in the aperiodic arrangement of the secondary structure elements (SSEs), which we deduce by calculating the nematic order parameter, $P_{2}$. We find that the folded structures are nematic droplets with a broad distribution of $P_{2}$. We argue that a non-zero value of $P_{2}$, leads to an arrangement of the SSEs that can resist external forces, which is a requirement for allosteric proteins. Such proteins, which resist mechanical forces in some regions while being flexible in others, transmit signals from one region of the protein to another (action at a distance) in response to binding of ligands (oxygen, ATP, or other small molecules).
\end{abstract}

Citation: Chakraborty, D.; Mugnai, M.L.; Thirumalai, D. On the Emergence of Orientational Order in Folded Proteins with Implications for Allostery. Symmetry 2021, 13, 770. https://doi.org/10.3390/sym13050770

Academic Editors: Theo Odijk,

Paul van der Schoot and

Abhijit Chakraborty

Received: 2 March 2021

Accepted: 20 April 2021

Published: 29 April 2021

Publisher's Note: MDPI stays neutral with regard to jurisdictional claims in published maps and institutional affiliations.

Copyright: (c) 2021 by the authors. Licensee MDPI, Basel, Switzerland. This article is an open access article distributed under the terms and conditions of the Creative Commons Attribution (CC BY) license (https:// creativecommons.org/licenses/by/ $4.0 /)$.
Keywords: secondary structure elements; nematic order parameter; allostery

\section{Introduction}

In his prescient and magnificent monograph, What is Life?, Schrödinger [1] states that, and we quote "...the most essential part of a living cell-the chromosome fibre may suitably be called an aperiodic crystal". He goes on to add that the difference between the well-known periodic crystal and and an aperiodic crystal is "...the same kind as that between an ordinary wallpaper in which the same pattern is repeated again and again in regular periodicity and a masterpiece of embroidery, say Raphael tapestry, which shows no dull repetition, but an elaborate, coherent, meaningful design traced by the great master", and concludes that an aperiodic crystal is the "material carrier of life". Of course, similar comments are applicable to proteins, the workhorses of the cellular machinery, which carry out a diverse range of functional roles, by the complex organization of their three-dimensional structures. Because proteins can be crystallized, they diffract X-rays, but their arrangement cannot be classified using a unit cell, which is used for describing the pattern repeated in the entire crystal. More importantly, a single folded globular protein (or a complex) does have some kind of order (certainly discernible to the eye) but is not periodic, and hence qualifies as an aperiodic crystal. The presence of well-defined scaffolds within the protein architecture does not imply structural rigidity, and it is reasonable to suggest that nearly all proteins can access evolutionarily tuned deformation modes [2-4] in order to carry out their functions. In addition, it has been shown that the coexistence of symmetrically arranged secondary structure elements (SSEs) with flexible regions within a single protein molecule could also enhance the multiplicity of folding pathways [5]. Thus, the aperiodic folds of proteins could have multiple functional consequences. Understanding the general rules underlying the packing of SSEs [6-8] is also important from the perspective of structure prediction.

In a pioneering study [6], Levitt and Chothia made far-reaching conclusions by analyzing the organization of the SSEs in 31 protein structures. They suggested that $\beta$-strands, 
which are adjacent in the sequence are ordered by forming hydrogen bonds or van der Waals contacts. It was also realized that such SSEs could form diffusing folding units, which then consolidate the native structure [6]. These ideas have been exploited in recent times in de novo protein design [9-11], the rationalization that geometry and symmetry considerations alone restrict the formation of native folds [12], and in exploring the so-called 'dark matter' of the protein-universe [13,14]. In a most insightful article, Wolynes [15] recognized explicitly the importance of aperiodic crystalline nature of proteins, and the resulting approximate symmetry. Linking these ideas to the principle that the folded state should be minimally frustrated [16], he suggested a connection between folding dynamics, approximate symmetry, and the underlying energy landscape. Thus, not only structures, but functional protein dynamics, are related to the aperiodic nature of the folded state of proteins.

An intriguing function of both single folded proteins or oligomers is allosteric signaling (action at a distance), which involves signal propagation through an ordered protein, usually in response to the binding of a ligand [17-20]. An excellent monograph on allostery from a unique perspective has recently been published by Rob Phillips [21]. Allostery requires the ability to impact chemistry occurring at sites beyond the reach of electrostatic forces between atoms-in other words, it is an emergent property [22] of the protein structure itself. The best studied example is the structural transitions that occur in Hemoglobin [23,24], when an oxygen molecule binds to the heme group. Elsewhere [25], we have argued that allosteric proteins (monomers, multi-domain proteins, or molecular machines) must be aperiodic [1]. This means that these biological molecules must have an approximate symmetry, implying that at least a portion of the complex must be rigid [22] in order to resist mechanical deformations, but not overly so, thus enabling the execution of those chemical and mechanical functions, which rely upon conformational flexibility. Without an approximate symmetry, allosteric transitions would not occur. Thus, there has to be a symmetry breaking transition when proteins fold from the unfolded high symmetry state. The symmetry requirement for allosteric transitions is the key premise of the much celebrated Monod-Wyman-Changeux (MWC) model [26], which explains not only the allosteric mechanism of Hemoglobin [24], but also various types of signal transduction [27]. However, not all allosteric transitions satisfy the symmetry requirements imposed by the MWC theory [18]. In this context, the Koshland-Némethy-Filmer (KNF) model [28] has found numerous applications, particularly in examples of 'induced fit' allostery [29] and negative cooperativity [30]. An alternative viewpoint of protein allostery, inspired by the model of Cooper and Dryden [31], has recently come to the forefront. In this picture, allosteric transitions can occur due to an alteration in the thermal fluctuations (or dynamics) around the native state as a result of ligand binding, and do not require substantial conformational changes [32-34]. In all these scenarios, structures with approximate symmetry are paramount.

Here, we suggest that there is a breakage of orientational symmetry when proteins fold. This is manifested in the emergence of nematic order, in some instances only weakly so, which is sufficient for many proteins to behave as allosteric systems. Because allostery involves accessing alternate aperiodic states, which are excitations around the ground state, both the ground and excited states must have similar, but not necessarily identical symmetry. For biological viability, the free energy gap between the ground and the excited state cannot be too large [25], which also implies that the structured regions cannot be very rigid. We show, by analyzing the structures of a number of globular proteins and oligomeric assemblies, that they have non-zero values of the nematic order parameter, $P_{2}$. There are large variations in $P_{2}$. The values of $P_{2}$ for allosteric proteins are non-zero but not close to unity. As the magnitude of the order parameter measures the degree of the broken symmetry [22], intermediate values of the order parameter reflect the delicate balance between rigidity and conformational flexibility. 


\section{Methods}

\subsection{Protein Structures}

Database of globular proteins: To build a representative database of globular proteins, we considered only those sequences from the PDB database that meet the following criteria: (1) mutual sequence identity of no more than $25 \%$; (2) at least 40 amino acids in length; (3) structural resolution of at least $3 \AA$. The list of such sequences was determined using the PISCES web-server [35]. Any sequence that is known to form a membrane protein has a corresponding entry in either the PDBTM [36], mpstruc [37], or the OPM [38] database, and is excluded from our analysis. We do so out of an abundance of caution because, unlike globular proteins, membrane proteins are not evolved to be optimally compact, and the membrane itself plays an active role in their folding and assembly.

Subset of allosteric domains: A non-redundant subset of experimentally determined allosteric domains was obtained from the "Core set" of the ASBench dataset maintained by Zhang and coworkers [39,40]. The ASBench suite consists of 235 unique allosteric sites, predominantly found in E. coli. and humans. These allosteric sites are distributed among diverse protein families, including transferases, hydrolases, oxidoreductases, transcription factors, and lyases [39].

\subsection{Data Analysis}

Residue-specific secondary structure: The residue-wise secondary structure of the globular proteins in the curated database was determined using the DSSP algorithm [41] implemented within the mdtraj program [42]. For simplicity, we assigned the secondary structure based on a three-class system, which identifies each residue as being in helix $(\mathrm{H})$, $\beta$-sheet (E), or in coil/turn configuration (C). Based on the secondary structure content, we segregate all the proteins in the database into three types: if the overall $\alpha$-helicity of a protein structure exceeds $20 \%$, and the $\beta$-content is less than $10 \%$, the protein is classified as $\alpha$-rich; if the net $\beta$-content is greater than $20 \%$, and the $\alpha$-helix content is less than $10 \%$, the protein is considered to be $\beta$-rich; if both the $\alpha$-helix and the $\beta$-content exceeds $12 \%$, then we classify the protein as $\alpha+\beta$. We note that the cutoffs employed for determining the different protein structural classes are quite robust, and similar statistics were obtained for variations within a reasonable range of these cutoff values. To further distinguish among the different topologies adopted by the three protein classes, we use the CATH classification of proteins [43] as a guide.

Ordering of secondary structure elements (SSEs): To probe the nematic ordering of secondary structure elements (SSE) within the protein fold, we compute the nematic order parameter using the WORDOM package $[44,45]$. The traceless second-rank tensor $Q$ is calculated from molecular vectors, $\vec{w}_{i}$ as $[46,47]$ :

$$
Q_{m n}=\frac{1}{N_{s}} \sum_{i=1}^{N_{s}} \frac{3}{2} w_{i m} w_{i n}-\frac{1}{2} \delta_{m n}
$$

In Equation (1), indices $m$ and $n$ denote the $x, y$, and $z$-directions, and $\vec{w}_{i} \mathrm{~s}$ are the molecular vectors connecting the $C_{\alpha}$ atoms located at the terminii of secondary structure elements, such as the $\alpha$-helix, or the $\beta$-sheet; $N_{S}$ denotes the total number of $\alpha$-helices and $\beta$-sheets within the protein fold; these secondary structure elements (SSEs) can be part of a single or multiple different chains. Diagonalization of $Q$ yields the nematic director, $d$, which is the eigenvector associated with the maximum non-zero eigenvalue [46,48]. as [45]:

From $Q$ (Equation (1)), and the director $\vec{d}$, the nematic order parameter, $P_{2}$, is computed

$$
P_{2}=\vec{d} \cdot Q \cdot \vec{d}
$$

For a near perfect ordering of SSEs, $P_{2} \approx 1$, while $P_{2} \approx 0$ for isotropic arrangements.

A diagrammatic illustration of the vectors, $\vec{w}_{i}$, for a $\alpha+\beta$ protein is shown in Figure 1 . Here, $\vec{h}$ denotes a $\vec{w}_{i}$ vector directed along the axis of the $\alpha$-helical segment, and $\vec{b}$ is a $\vec{w}_{i}$ vector directed along the an extended $\beta$-sheet in the protein. 


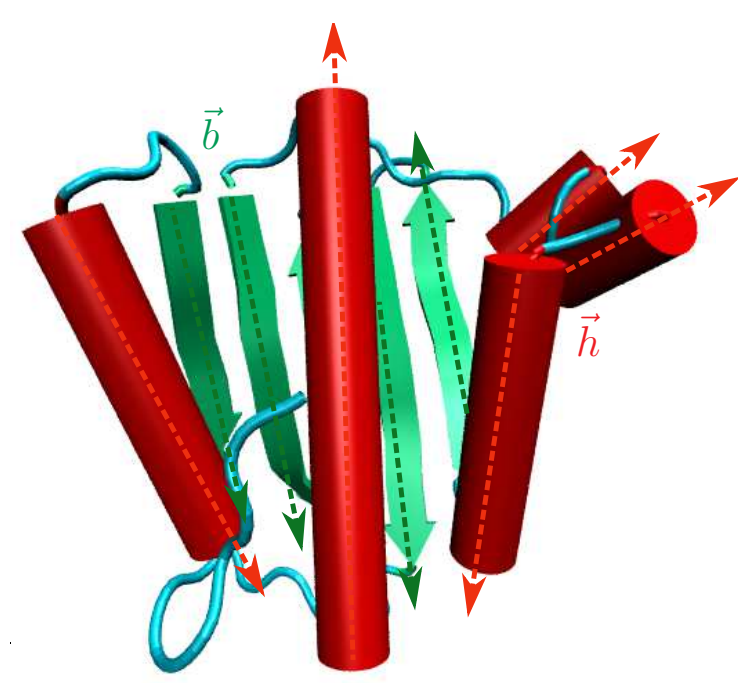

Figure 1. An illustration of the vectors, $\vec{w}_{i}$, that are used to compute the nematic order parameter, $P_{2}$ (see Equation (2)), for a $\alpha+\beta$ protein. The protein is shown in a cartoon representation, with the $\alpha$-helices depicted as red cylinders, and the extended $\beta$-sheets colored green. The irregular coils/turns connecting the different secondary structure elements (SSEs) are in cyan. The vectors (for example, $\vec{h}$ ) connecting the $C_{\alpha}$ atoms located at the termini of $\alpha$-helices are depicted as red arrows. Green arrows denote the vectors (for example, $\vec{b}$ ) connecting the $C_{\alpha}$ atoms located at the termini of extended $\beta$-sheets.

To test the statistical independence of different $P_{2}$ distributions, we carried out the Pearson $\chi^{2}$ test for homogeneity, as implemented within the scipy module.

\section{Results}

Single- and multi-chain proteins have different organization of secondary structural elements (SSEs): The distribution of the nematic order parameter, $P_{2}$, for the subset of single- and multi-chain globular proteins that meet the structural and sequence-identity criteria (see Methods), are shown in Figure 2a. The $P_{2}$ distribution for the multi-chain proteins peaks at relatively lower values compared to the single-chain proteins, due to the additional orientational degree of freedom dictating the relative arrangement in space of different monomeric units. In the aperiodic states, $P_{2} \neq 0$, implying that the ordered states are indeed nematic droplets in which orientational symmetry is broken. The relatively higher probability density in the region, $0.4 \leq P_{2} \leq 0.7$, for single-chain proteins, further indicates that they adopt more folds/topologies commensurate with intermediate ordering of the SSEs. The multi-chain $P_{2}$ distribution, on the other hand, features slightly enhanced probabilities in the tail region, beyond $P_{2} \approx 0.8$, suggesting that highly ordered assemblies (with only few packing defects) are more likely to result from oligomerization, rather than self-association of multiple SSEs within single-chains.

Additional insights into the different folds/topologies that are adopted by singleand multi-chain proteins can be obtained from the secondary-structure dependent $P_{2}$ distributions, shown in Figure 2b,c. We classify all proteins in the database into three broad classes: $\alpha$-rich, $\beta$-rich, and $\alpha+\beta$ (see Methods). Among $\alpha$-rich proteins, the ordering of SSEs is maximized within helical bundles and coiled-coils, featuring the helix-loop-helix type topology. A representative example of this fold includes the seven-helix bundle periplasmic sensor domain of the TorS receptors found in E. coli. (Figure 3). For singlechain $\beta$-rich proteins, the probability density at $P_{2} \geq 0.8$ is lower than that for the $\alpha$-rich counterparts, suggesting that the alignment of SSEs relative to one another is somewhat less efficient. The ordering of SSEs within single-chain $\beta$-rich proteins is maximized primarily in sandwich domains having the immunoglobin fold (involved in passive muscle elasticity), although some highly ordered structures also exhibit other topologies, such as jelly rolls, and $\beta$-prisms. A few representative examples of such folds are shown in Figure 3. 

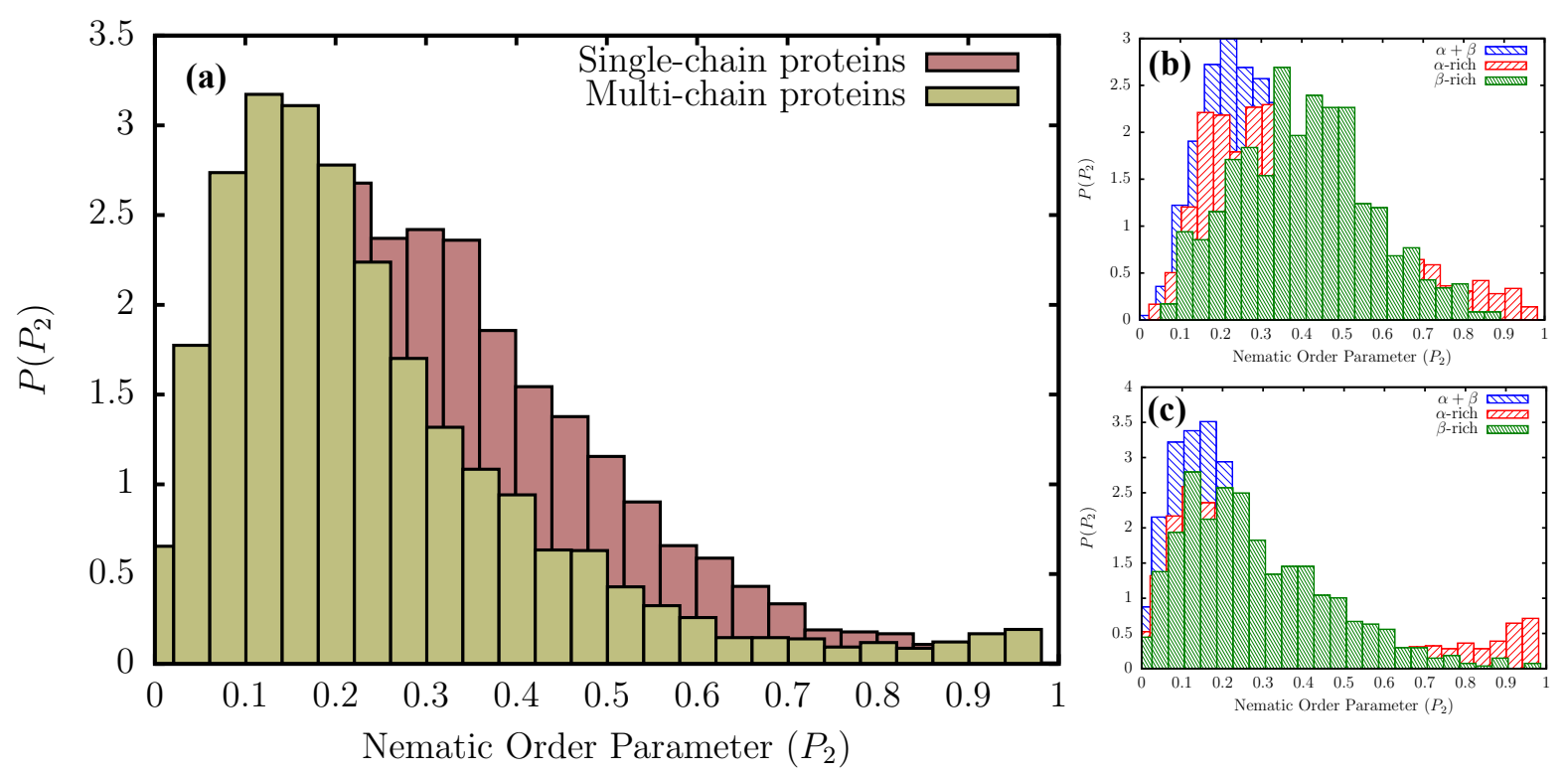

Figure 2. (a) Distribution of the nematic order parameter, $P_{2}$, for single- and multi-chain globular proteins present in the database constructed using the PISCES web-server. The secondary-structure dependent $P_{2}$ distributions in singleand multi-chain proteins are shown in $(\mathbf{b}, \mathbf{c})$, respectively. As outlined in the Methods section, the residue-dependent secondary-structures were computed using the DSSP algorithm.

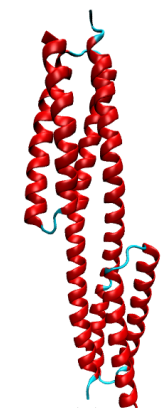

(a)

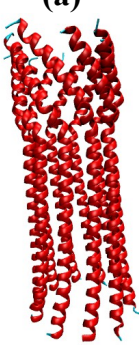

(f)

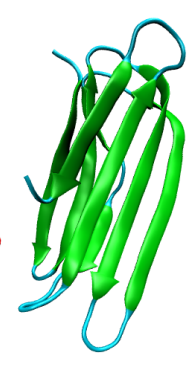

(b)

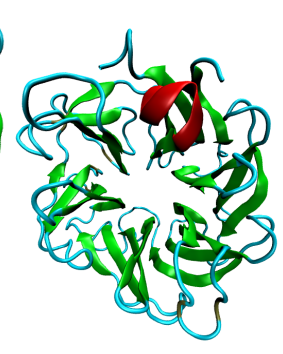

(c)

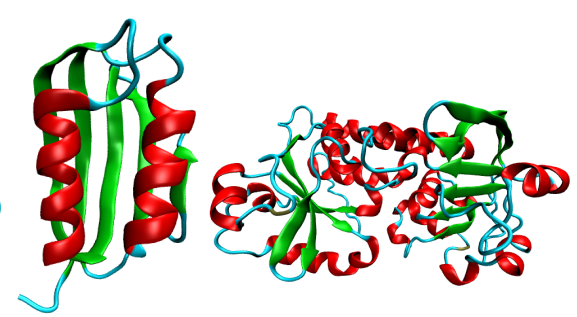

(d)

(e)

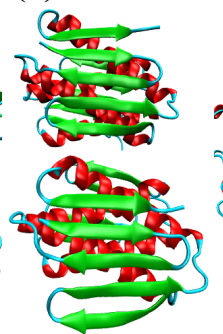

(i)

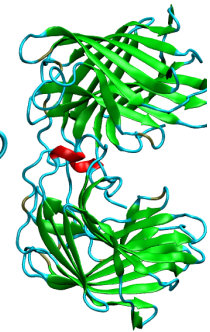

(h)

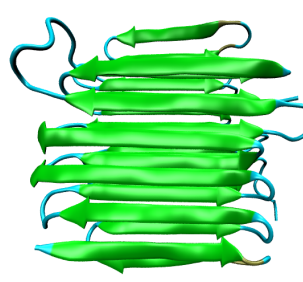

(g)

Figure 3. Some representative protein folds found among proteins in the PDB database. (a) sevenhelix bundle periplasmic sensor domain of the TorS receptors (PDB ID: 3I9Y); (b) Immunoglobin-like fold (PDB ID: 2XSK) present in the E. coli. curli protein; (c) 6-blade $\beta$-propeller (PDB ID: 3A72) found within a high-resolution crystal structure of Penicillium chrysogenum alpha-L-arabinase in complex with arabinobiose; (d) $\alpha \beta$ plait (PDB ID: 6CBU) topology found within an immunogen; (e) Rossmann fold (PDB ID: 4LJS) present in a periplasmic binding protein; (f) central domain of bacteriophage phiX174 H protein formed by the assembly of ten $\alpha$-helices (PDB ID: 4JPN); (g) 2solenoid topology found in antifreeze proteins (PDB ID: 4DT5); (h) jelly-roll (PDB ID: 4HFS) found in a protein from Bacilus subtilis subsp. subtilis str. 168. (i) $\alpha \beta$-plait (PDB ID: 1S12) within the protein TM1457 from Thermotoga maritama; (j) TIM barrel (PDB ID: 3CBW) found in the YdhT protein from Bacilus subtilis. 
In contrast to the other two protein classes, single-chain $\beta$-proteins seem to form a large number of folds characterized by intermediate ordering of the SSEs $\left(P_{2}\right.$ in between 0.4 and 0.5 ). We find that these folds largely have the sandwich-type architecture, with either the immunoglobin or the jelly roll-type topology. Some proteins also adopt the more exotic $\beta$-propeller topology, with the number of blades varying between five and eight (Figure 3 ). The difference in the $P_{2}$ distributions between single-chain and multi-chain proteins can largely be explained by this ability of single-chain $\beta$-rich proteins to form such diverse folds, exhibiting intermediate ordering of SSEs. As is evident from Figure $2 b, \alpha+\beta$ proteins are also less likely to form folds that maximize the ordering of the SSEs $\left(P_{2} \geq 0.8\right)$, as compared to $\alpha$-rich proteins. Nonetheless, we find few topologies, such as $\alpha \beta$-plaits, and leucine-rich repeats, where SSEs could be maximally aligned (Figure 3 ). The peak of the $P_{2}$ distribution for $\alpha+\beta$ proteins occurs at $\approx 0.2$, consistent with a large number of architectures found in the database, such as the two-layer sandwich, three-layer aba sandwich, $\alpha \beta$-complex, and the $\alpha \beta$-barrel. In particular, we find that the Rossmann fold (three-layer aba sandwich), $\alpha \beta$-plaits, and TIM barrels ( $\alpha \beta$-barrel) are particularly favored (Figure 3).

Similar to their single-chain counterparts, multi-chain $\alpha$-rich proteins also form a larger number of orientationally ordered structures, compared to the other two protein classes, by exploiting the helix-bundle architecture, as in the case of the central domain of bacteriophage phiX174 H protein. Multi-chain $\beta$-proteins maximize the ordering of SSEs through a diverse range of folds including aligned prisms, jelly rolls, solenoids, and immunoglobinlike sandwich architectures. However, unlike their single-chain counterparts, the subset of multi-chain $\beta$-proteins also includes highly ordered structures $\left(P_{2} \geq 0.9\right)$, such as amyloid fibrils, and antifreeze proteins exhibiting the 2-solenoid topology (Figure 3). Interestingly, multi-chain $\beta$ proteins do not adopt as many folds of intermediate order, suggesting that additional symmetry requirements could impose constraints on the formation of such topologies (Figure 2c). The $P_{2}$ distribution for multi-chain $\alpha+\beta$ proteins is quite similar to that for single-chains. A large number of the structures exhibit intermediate ordering of the SSEs (peak at $\approx 0.2$ ), and belong to the Rossmann fold, $\alpha \beta$-plait, or the TIM barrel superfamily. In addition, like single-chains, multi-chain $\alpha+\beta$ proteins also primarily exploit the $\alpha \beta$-plait topology to maximally align SSEs (Figure 3 ).

Overall, we find that, for most single- and multi-chain proteins found in the PDB database, the arrangement of SSEs exhibits orientational order. It may be argued that nematic order is weak because $P_{2}$ values are not too close to unity. Only a few topologies are exploited to form highly symmetrical or ordered structures.

Allosteric domains display weak nematic order: In most proteins, allostery is modulated through the concerted motions of structural elements, such as strands and helices, orchestrated by flexible loops or hinges. One of the key requirements in allostery is that certain regions of the protein must be stiff enough to resist mechanical stress, and yet the overall architecture should be flexible enough to accommodate subtle changes in conformation [25]. Therefore, understanding the precise ordering of SSEs, as well as fold preferences within allosteric domains becomes crucial.

We find that, in contrast to protein structures culled using the PISCES web-server, the nematic order parameter, $P_{2}$, for allosteric domains does not exceed $\approx 0.5$ (Figure 4 ). Therefore, the arrangement of SSEs within allosteric domains cannot be overtly stiff, like in ordered assemblies, such as fibers and helix bundles, and must be flexible enough to access the excited state in order to accommodate structural perturbations. In a recent study, Xie and Lai [49] argued that allosteric domains have a preference for certain topologies like the Rossmann fold, while certain folds like the immunoglobin-like $\beta$-sandwiches are disfavored. Our results support such a viewpoint because we find that the distributions of the nematic order parameter for allosteric domains lack the long tails at high $P_{2}$ values, which in the case of single-chain $\beta$-rich proteins primarily result from immunoglobulin-like folds that can withstand large mechanical forces. Furthermore, the enhanced probabilities at $P_{2} \approx 0.2$ (Figure 4 ) also reflect the preference of allosteric domains for Rossmann folds [49]. 


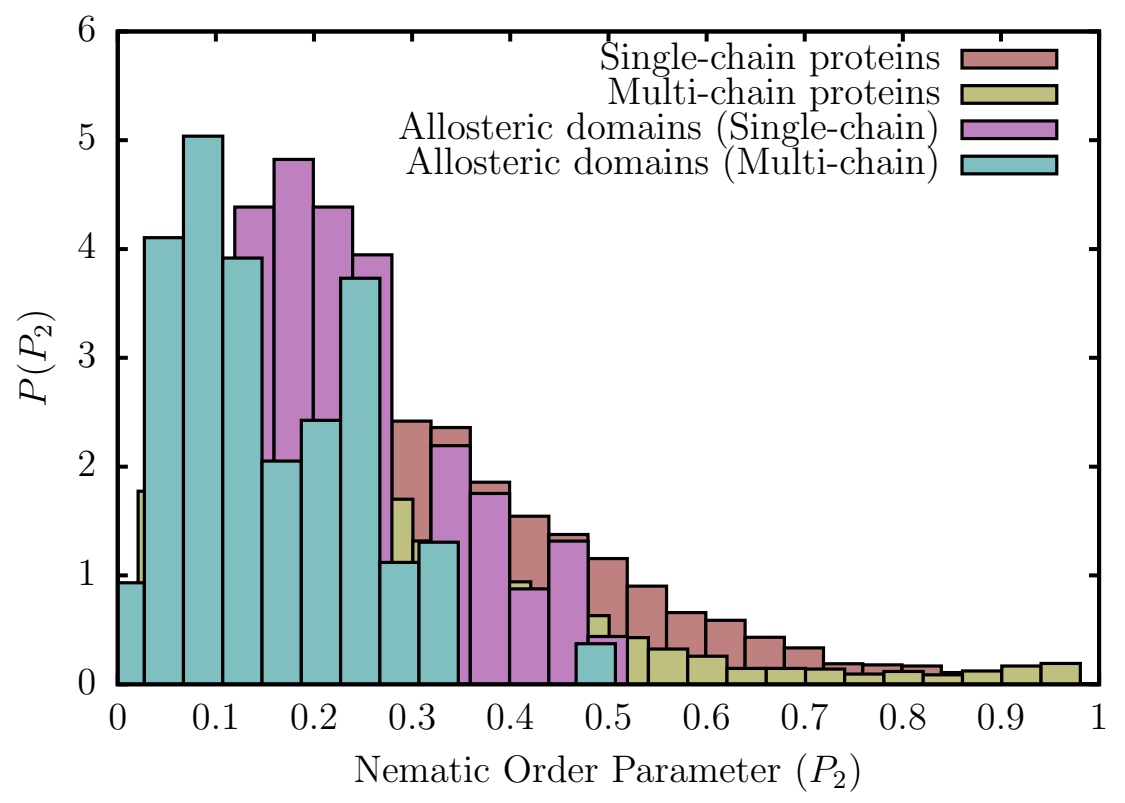

Figure 4. Distribution of the nematic order parameter, $P_{2}$, for single- and multi-chain proteins culled using the PISCES web-server, and allosteric domains present in the ASBench database. For allosteric domains, the nematic order parameter, $P_{2}$, has an upper bound of $\approx 0.5$, and the corresponding distributions lack long tails at high $P_{2}$ values (resulting from highly ordered assemblies). In case of single-chain proteins, the Pearson $\chi^{2}$ test for homogeneity yields a $p$-value of 0.02 , indicating that the distributions computed from the ASBENCH and PISCES databases are statistically independent. The same is true for multi-chain proteins, for which a $p$-value of 0.0001 is obtained.

Among single-chain allosteric domains included within the ASBench database, the human kinesin spindle protein (also known as Eg5) exhibits the maximal ordering of secondary structural elements, with a $P_{2}$ value of $\approx 0.5$ (Figure $5 \mathrm{a}$ ). Within the kinesin superfamily, Eg5 is one of the most advanced drug targets, and most designed inhibitors, such as ispinesib, specifically bind to an allosteric pocket located around $10 \AA$ away from the ATP binding site $[50,51]$. Recent evidence $[51,52]$ suggests that the binding of ispinesib and other drugs to the allosteric pocket of Eg5 only induces minimal structural changes in the arrangement of the SSEs, and much of the perturbation is accommodated by the flexible loop region. Another prototypical single-chain allosteric domain is found in the protein kinase A (PKA) regulatory subunit of yeast [53]. The enzyme consists of two cyclic adenosine monophosphate (cAMP) binding domains (Figure 5b), and the unique interface formed between the two domains modulates the allosteric mechanism underlying PKA activation [53]. Substantial structural changes, which occur at the interface due to cAMP binding, are primarily driven by the movements of the helical subunits within the cyclic nucleotide binding (CNB) domains. Thus, in contrast to Eg5, allosteric transitions in this protein require a very labile network of SSEs, associated with low nematic order. We find that this is precisely the case, and the $P_{2}$ value for the yeast regulatory subunit is only around 0.15 .

Within the subset of multi-chain allosteric domains, the regulatory domain of Leptospira interrogans citramalate synthase (LiCMS) is associated with the highest value of $P_{2}(\approx 0.5)$. The functional form of LiCMS is a homodimer (Figure $5 \mathrm{c}$ ), and it is involved in the catalysis of the first step of the isoleucine biosynthesis pathway in L. interrogans. This reaction is modulated by a feedback inhibition mechanism, mediated by the binding of isoleucine to the allosteric site at the dimer interface [54]. Structural and biochemical evidence strongly suggests that the inhibition of LiCMS is likely to occur via interdomain communication between the regulatory and catalytic domains, rather than large-scale rearrangements in the quaternary structure of the protein [54]. This scenario for allosteric regulation seems consistent with the somewhat rigid packing of SSEs found in this protein. 
As a final example, we consider the well-studied GroEL-GroES complex [55], an ATP-fueled nanomachine, which aids in protein folding, by undergoing a series of complex allosteric transitions [56,57]. The different rings of the GroEL-GroES complex move in a concerted fashion, with the inter-ring motions being anticorrelated, resulting in a form of nested cooperativity [58]. It is evident that intra- and inter-subunit rearrangements underlying such complex allostery can only be attained through an arrangement of structural elements, which can be disrupted by moderate strain. Indeed, in the GroEL-GroES complex, the extent of nematic order is small $\left(P_{2} \approx 0.06\right)$.

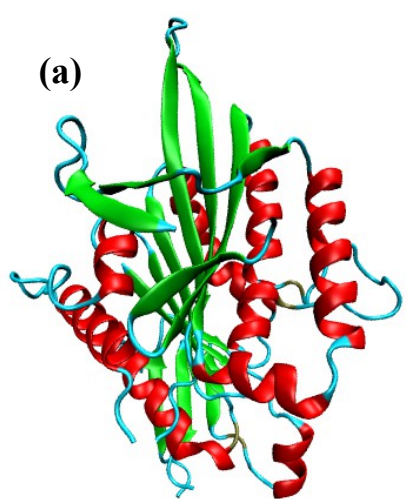

(c)

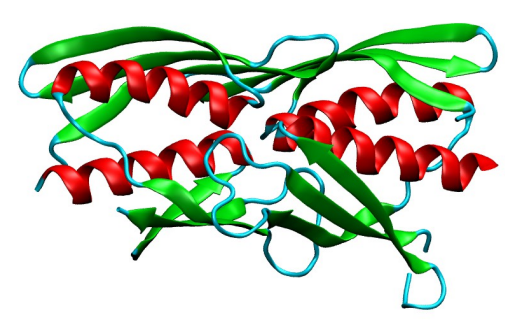

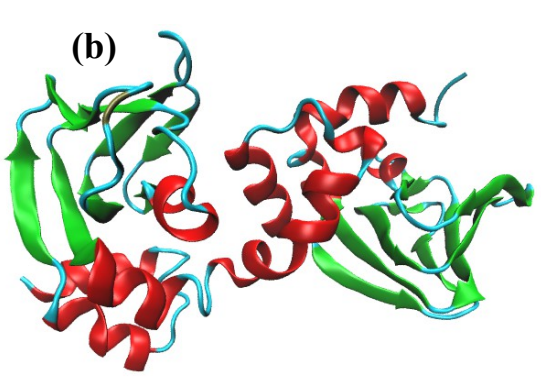

(d)

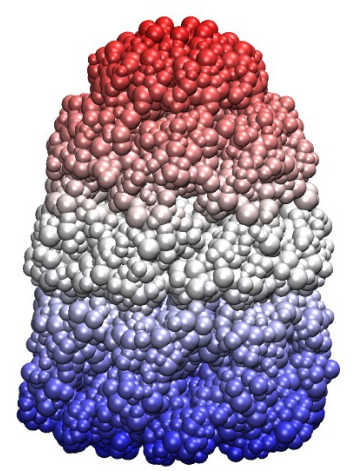

Figure 5. Some representative examples of allosteric domains. (a) human kinesin spindle protein, Eg5 (PDB ID: 3ZCW); (b) PKA regulatory subunit of yeast (PDB ID: 3OF1); (c) regulatory domain of LiCMS (PDB ID: 3F6G); (d) bullet shaped GroEL-GroES complex (PDB ID: 1PCQ).

The examples above suggest that the extent of nematic order found in allosteric proteins has been tuned to carry out a specific function. While allosteric inhibition could be accommodated by a rigid arrangement of SSEs, complex allosteric transitions require a somewhat flexible network of structural elements that are arranged in an aperiodic manner, but responds to stresses readily. It is worth remembering that allostery requires that the two states (usually referred to as $T$ and $R$ ) be readily accessible upon ligand binding (oxygen to Hemoglobin $(\mathrm{Hb})$ for example). In the absence of the ligand, the $R$ state would be an excited state separated from the $T$ state by a free energy gap. During the $T \rightarrow R$ transition, although the overall symmetry might be preserved, the extent of order often changes, reflecting the effect of the ligand. Indeed, in a family of Hemoglobins, we find that $P_{2}$ is greater in the $R$ state compared to the $T$ state. Thus, the strict preservation of the approximate symmetry in the ground state is neither required nor is observed.

Nematic order in different allostery models: The MWC and KNF phenomenological models $[26,28]$ were the earliest ones to describe protein allostery, and correspond to two extreme scenarios of ligand binding (namely, 'conformational selection' and 'induced fit'), in the energy landscape picture. A third possibility was raised by Dryden and Cooper [31], wherein allostery could occur without significant conformational change. In reality, proteins may accomplish their allosteric functions through a combination of these mechanisms, and a clear-cut distinction between them (for example, the MWC and KNF scenarios) may not always be feasible, although a recipe based on Hill coefficients has recently been suggested by Horovitz and coworkers [59]. We consider a representative example for each allostery 
model, and show that the nematic order, $P_{2}$ could be different for the ligand-free and ligand-bound states, as in the case of Hemoglobin.

For L-lactate Dehydrogenase (LDH), an archetypal example of MWC-type allostery (Figure 6) [60], the $R$ state is associated with a marginally higher value of $P_{2}$ as compared to the $T$ state, implying that the protein becomes somewhat rigid upon ligand binding [61]. The change in $P_{2}$ is more dramatic in the case of guanylate kinase (GK), which accomplishes its allosteric function through an 'induced fit' (KNF-type) mechanism [62], and undergoes a large conformational rearrangement upon binding of guanosine monophosphate (GMP). Finally, we consider the PDZ3 domain, which is known to exhibit dynamic allostery $[63,64]$. As expected, the $R$ and $T$ states exhibit only slightly different values of $P_{2}$, which could be an example of a CD-type mechanism (Figure 6).

Although it is tempting to deduce the plausible allosteric mechanisms, only on the basis of the $P_{2}$ values for the end-states, a note of caution is in order. We stress that the knowledge of intermediate states is essential. In this regard, a systematic comparison of how $P_{2}$ evolves along the pathways connecting the ligand-free and ligand-bound states could provide a means to distinguish between the different allosteric models. We envisage that the nematic order would change more abruptly along a KNF-type pathway, as compared to a MWC-type mechanism, while, for a CD-type mechanism, which proceeds via little or no conformational change, the deviation would be minimal.

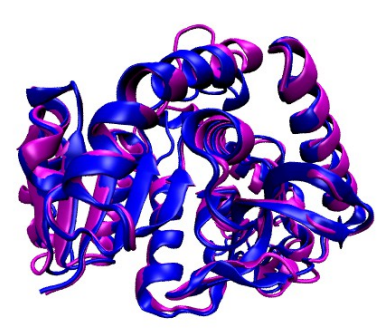

(a)

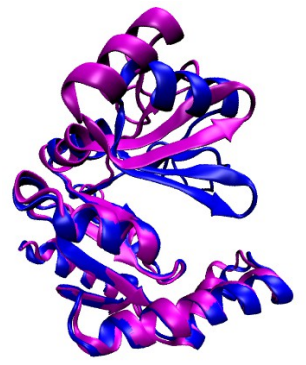

(b)

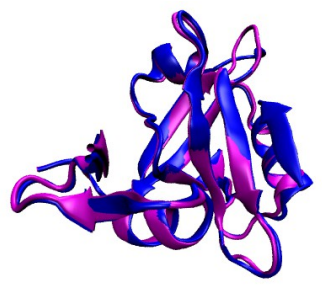

(c)

Figure 6. Representative examples of proteins exhibiting distinct allosteric mechanisms. The ligandfree state is shown in magenta, and the ligand-bound state is shown in blue. (a) L-lactate Dehydrogenase (PDB ID: 1LTH) is an example of an enzyme exhibiting MWC-type allostery. The $P_{2}$ value for the $T$ state is 0.25 , and for the $R$ state is 0.28 ; (b) Guanylate Kinase depicted in the ligand-free (PDB ID: 1EX6) and ligand-bound (PDB ID: 1EX7) forms. The ligand-free form has a $P_{2}$ value of 0.38 , and the ligand-bound form has a $P_{2}$ value of $\approx 0.56$. In this example, the ligand binding occurs via an induced-fit (KNF-type) mechanism, involving substantial conformational change; (c) the PDZ3 domain, which exhibits dynamic allostery. The ligand-free (PDB ID: 1BFE) and ligand-bound (PDB ID: 1BE9) states are structurally very similar, and are associated with $P_{2}$ values of 0.13 and 0.15 , respectively.

The role of packing defects in allostery: The presence of packing defects within folded protein architectures could also affect their ability to undergo conformational switch, and carry out allosteric functions. In fact, work dating back to the late 1980s shows that engineering a packing defect in an enzyme, by means of a point mutation, could induce allosteric behavior [65]. A recent study [66] also underscores the importance of packing defects in the allosteric binding of a feedback inhibitor to the Human UDP-Glucose Dehdyrogenase via an induced fit mechanism.

\section{Conclusions}

The precise arrangement of secondary structure elements within protein structures holds important cues for understanding their potential as an aggregation seed, allosteric scaffold, or a therapeutic target, not to mention the designability of proteins and their folding. In this study, we assessed the organization of secondary structure elements for 
a subset of proteins found in the PDB database, in terms of the nematic order parameter, $P_{2}$, which has been traditionally exploited in the studies of anisotropic molecules that break orientational symmetry when closely packed. We find that the distributions of $P_{2}$ are sensitive to the number of polypeptide chains present in the protein molecule, as well as the overall secondary structure. The secondary structure elements are maximally aligned in highly ordered structures, such as helix-bundles, amyloid fibers, and antifreeze proteins. However, as indicated by the small to intermediate values of $P_{2}$, in most allosteric proteins, the arrangement of SSEs exhibits nematic order but coexists with other more flexible regions (loops for example). Such an arrangement, observed in diverse fold topologies, ensures that the aperiodically arranged structures can transmit allosteric signals over the length of the complex, while the flexible regions can rearrange to readily access the excited states for carrying out specific functions.

In allosteric domains, the SSEs cannot be maximally aligned (i.e., $P_{2} \approx 1$ ) because that would make the structure too rigid. The extent of nematic ordering is functionally tuned, and depends on the precise structural requirements underlying the allosteric transitions. Based on the large overlap between the $P_{2}$ distributions near the peak regions for proteins in the PISCES database, and the ASBench subset, we speculate that many more non-fibrous proteins than currently known could potentially be allosteric under suitable conditions.

Author Contributions: D.C., M.L.M., and D.T. designed research; D.C. performed research and analyzed data; and D.C., M.L.M., and D.T. wrote the manuscript. All authors have read and agreed to the published version of the manuscript.

Funding: This work was supported by the National Science Foundation (CHE19-00093), and by the Collie-Welch Chair administered through the Welch Foundation (F-0019).

Institutional Review Board Statement: Not applicable.

Informed Consent Statement: Not applicable.

Data Availability Statement: The analysis scripts and three-dimensional coordinates of the protein structures are available from the authors upon reasonable request.

Acknowledgments: We are grateful to Bill Eaton, Gilad Haran, Hagen Hoffmann, and Amnon Horovitz for useful comments.

Conflicts of Interest: The authors declare no conflict of interest.

\section{References}

1. Schrodinger, E. What Is Life; University Press: Cambridge, UK, 1943.

2. Falke, J.J. Enzymology: A moving story. Science 2002, 295, 1480-1481. [CrossRef] [PubMed]

3. Fraunfelder, H.; Mcmohan, B.H.; Austin, R.H.; Chu, K.; Groves, J.T. The role of structure, energy landscape, dynamics, and allostery in the enzymatic function of myoglobin. Proc. Natl. Acad. Sci. USA 2001, 98, 2370-2374. [CrossRef] [PubMed]

4. Fraunfelder, H.; Sligar, S.G.; Wolynes, P.G. The energy landscapes and motions of proteins. Science 1991, 254, 1598-1603. [CrossRef] [PubMed]

5. Klimov, D.K.; Thirumalai, D. Symmetric Connectivity of Secondary Structure Elements Enhances the Diversity of Folding Pathways. J. Mol. Biol. 2005, 353, 1171-1186. [CrossRef]

6. Levitt, M.; Chothia, C. Structural patterns in globular proteins. Nature 1976, 26, 552-558. [CrossRef] [PubMed]

7. Chothia, C.; Levitt, M.; Richardson, D. Structure of proteins: Packing of alpha-helices and pleated sheets. Proc. Natl. Acad. Sci. USA 1977, 74, 4130-4134. [CrossRef]

8. Chothia, C. Principles that determine the structure of proteins. Annu. Rev. Biochem. 1984, 53, 537-572. [CrossRef]

9. Minami, S.; Sawada, K.; Chikenji, G. How a spatial arrangement of secondary structure elements is dispersed in the universe of protein folds. PLoS ONE 2014, 9, e107959. [CrossRef]

10. Woolfson, D.N.; Bartlett, G.J.; Burton, A.J.; Heal, J.W.; Nitsu, A.; Thomson, A.R.; Wood, C.W. De novo protein design: How do we expand the universe of possible protein structures. Curr. Opin. Struct. Biol. 2015, 33, 16-26. [CrossRef]

11. Huang, P.S.; Oberdorfer, G.; Xu, C.; Pei, X.; Nannenga, B.; Rogers, J.M.; DiMaio, F.; Gonen, T.; Luisi, B.; Baker, D. High thermodynamic stability of parametrically designed helical bundles. Science 2014, 346, 481-485. [CrossRef] [PubMed]

12. Hoang, T.X.; Trovato, A.; Seno, F.; Banavar, J.R.; Maritan, A. Geometry and symmetry presculpt the free-energy landscape of proteins. Proc. Natl. Acad. Sci. USA 2004, 101, 7960-7964. [CrossRef]

13. Levitt, M. Nature of the Protein Universe. Proc. Natl. Acad. Sci. USA 2009, 7, 11079-11084. [CrossRef] [PubMed] 
14. Perdigao, N.; Henrich, J.; Stolte, C.; Sabir, K.S.; Buckley, M.J.; Tarbor, B.; Signal, B.; Gloss, B.S.; Hammang, C.J.; Rost, B.; et al. Unexpected features of the dark proteome. Proc. Natl. Acad. Sci. USA 2015, 112, 15898-15903. [CrossRef]

15. Wolynes, P.G. Symmetry and energy landscapes of biomolecules. Proc. Natl. Acad. Sci. USA 1996, 93, 14249-14255. [CrossRef]

16. Onuchic, J.N.; Wolynes, P.G. Theory of protein folding. Curr. Opin. Struct. Biol. 2004, 14, 70-75. [CrossRef]

17. Wodak, S.J.; Paci, E.; Dokholyan, N.; Berezovsky, I.N.; Horovitz, A.; Li, J.; Hilser, V.J.; Bahar, I.; Karanicolas, J.; Stock, G.; et al. Allostery in Its Many Disguises: From Theory to Applications. Structure 2019, 27, 566-578. [CrossRef] [PubMed]

18. Cui, Q.; Karplus, M. Allostery and cooperativity revisited. Protein Sci. 2008, 17, 1295-1307. [CrossRef] [PubMed]

19. Campbell, E.; Kaltenbach, M.; Correy, G.J.; Carr, P.D.; Porebski, B.T.; Livingstone, E.K.; Afriat-Jurnou, L.; Buckle, A.M.; Weik, M.; Hollfelder, F.; et al. The role of protein dynamics in the evolution of new enzyme function. Nat. Chem. Biol. 2016, 12, 944-950. [CrossRef]

20. Sikosek, T.; Chan, H.S. Biophysics of protein evolution and evolutionary protein biophysics. J. R. Soc. Interface 2014, 11, 20140419. [CrossRef]

21. Phillips, R. The Molecular Switch: Signaling and Allostery; Princeton University Press: Princeton, NJ, USA, 2020.

22. Anderson, P.W. Basic Notions of Condensed Matter Physics; Westview Press: Boulder, CO, USA, 1997.

23. Yuan, Y.; Tam, M.F.; Simplaceanu, V.; Ho, C. New Look at Hemoglobin Allostery. Chem. Rev. 2015, 115, 1702-1724. [CrossRef]

24. Eaton, W.A.; Henry, E.R.; Hofrichter, J.; Mozzarelli, A. Is cooperative oxygen binding by hemoglobin really understood? Nat. Struct. Biol. 1999, 6, 351-358. [CrossRef] [PubMed]

25. Thirumalai, D.; Hyeon, C.; Zhuravlev, P.I.; Lorimer, G. Symmetry, Rigidity, and Allosteric Signaling: From Monomeric Proteins to Molecular Machines. Chem. Rev. 2019, 119, 6788-6821. [CrossRef] [PubMed]

26. Monod, J.; Wyman, J.; Changeux, J.P. On the nature of allosteric transitions: A plausible model. J. Mol. Biol. 1965, 12, 88-118. [CrossRef]

27. Changeux, J.P.; Edelstein, S.J. Allosteric Mechanisms of Signal Transduction. Science 2005, 308, 1424-1428. [CrossRef]

28. Koshland, D.E.; Nemethy, G.; Filmer, D. Comparison of Experimental Binding Data and Theoretical Models in Proteins Containing Subunits. Biochemistry 1966, 5, 365-385. [CrossRef]

29. Choy, M.S.; Li, Y.; Machado, L.E.S.F.; Kunze, M.B.A.; Connors, C.R.; Wei, X.; Lindorff-Larsen, K.; Page, R.; Peti, W. Conformational Rigidity and Protein Dynamics at Distinct Timescales Regulate PTP1B Activity and Allostery. Mol. Cell 2017, 65, 644-658. [CrossRef]

30. Henis, Y.I.; Levitki, A. Mechanism of negative cooperativity in glyceraldehyde-3-phosphate dehydrogenase deduced from ligand competition experiments. Proc. Natl. Acad. Sci. USA 1980, 77, 5055-5059. [CrossRef]

31. Cooper, A.; Dryden, D.T. Allostery without conformational change. A plausible model. Eur. Biophys. J. 1984, 11, 103-109. [CrossRef]

32. McLeish, T.C.B.; Rodgers, T.L.; Wilson, M.R. Allostery without conformation change: Modelling protein dynamics at multiple scales. Phys. Biol. 2013, 10, 056004. [CrossRef]

33. McLeish, T.C.B.; Cann, M.J.; Rodgers, T.L. Dynamic Transmission of Protein Allostery without Structural Change: Spatial Pathways or Global Modes? Biophys. J. 2015, 109, 1240-1250. [CrossRef]

34. McLeish, T.; Schaefer, C.; von der Heydt, A. The 'allosteron' model for entropic allostery of self-assembly. Phil. Trans. R. Soc. B 2018, 373, 20170186. [CrossRef] [PubMed]

35. Wang, G.; Dunbrack, R.L. PISCES: A protein sequence culling server. Bioinformatics 2003, 19, 1589-1591. [CrossRef]

36. Tusnady, G.E.; Dosztanyi, Z.; Simon, I. Transmembrane proteins in the Protein Data Bank: Identification and classification. Bioinformatics 2004, 20, 2964-2972. [CrossRef]

37. White, S.H. Biophysical dissection of membrane proteins. Nature 2009, 459, 344-346. [CrossRef]

38. Lomize, M.A.; Lomize, A.L.; Pogozheva, I.D.; Mosberg, H.I. OPM: Orientations of proteins in membranes database. Bioinformatics 2006, 22, 623-625. [CrossRef]

39. Huang, W.; Wang, G.; Shen, Q.; Liu, X.; Lu, S.; Geng, L.; Huang, Z.; Zhang, J. ASBench: Benchmarking sets for allosteric discovery. Bioinformatics 2015, 31, 2598-2600. [CrossRef]

40. Huang, Z.; Mou, L.; Shen, Q.; Lu, S.; Li, C.; Liu, X.; Wang, G.; Li, S.; Geng, L.; Liu, Y.; et al. ASD v2.0: Updated content and novel features focusing on allosteric regulation. Nucleic Acids Res. 2014, 42, D510-D516. [CrossRef] [PubMed]

41. Kabsch, W.; Sander, C. Dictionary of protein secondary structure: Pattern recognition of hydrogen-bonded and geometrical features. Biopolymers 1983, 22, 2577-2637. [CrossRef]

42. McGibbon, R.T.; Beauchamp, K.A.; Harrigan, M.P.; Klein, C.; Swails, J.M.; Hernández, C.X.; Schwantes, C.R.; Wang, L.P.; Lane, T.J.; Pande, V.S. MDTraj: A Modern Open Library for the Analysis of Molecular Dynamics Trajectories. Biophys. J. 2015, 109, 1528-1532. [CrossRef]

43. Dawson, N.L.; Lewis, T.E.; Das, S.; Lees, J.; Lee, D.; Ashford, P.; Orengo, C.; Sillitoe, I. CATH: An expanded resource to predict protein function through structure and sequence. Nucleic Acid. Res. 2019, 45, D289-D295. [CrossRef]

44. Seeber, M.; Felline, A.; Raimondi, F.; Muff, S.; Friedman, R.; Rao, F.; Calfisch, A.; Fanelli, F. Wordom: A user-friendly program for the analysis of molecular structures, trajectories, and free energy surfaces. J. Comp. Chem. 2011, 32, 1183-1194. [CrossRef] [PubMed]

45. Cecchini, M.; Rao, F.; Seeber, M.; Calfisch, A. Replica exchange molecular dynamics simulations of amyloid peptide aggregation. J. Chem. Phys. 2004, 121, 10748. [CrossRef] 
46. Wilson, M.R. Determination of Order Parameters in Realistic Atom-Based Models of Liquid Crystal Systems. J. Mol. Liq. 1996, 68, 23-31. [CrossRef]

47. Andrienko, D. Introduction to liquid crystals. J. Mol. Liq. 2018, 267, 520-541. [CrossRef]

48. Eppenga, R.; Frenkel, D. Monte Carlo study of the isotropic and nematic phases of infinitely thin hard platelets. Mol. Phys. 1984, 52, 1303-1334. [CrossRef]

49. Xie, J.; Lai, L. Protein topology and allostery. Curr. Opin. Struct. Biol. 2020, 62, 158-165. [CrossRef] [PubMed]

50. Ulaganathan, V.; Talapatra, S.K.; Rath, O.; Pannifer, A.; Hackney, D.D.; Kozielski, F. Structural Insights into a Unique Inhibitor Binding Pocket in Kinesin Spindle Protein. J. Am. Chem. Soc. 2013, 135, 2163-2272. [CrossRef] [PubMed]

51. Yokoyama, H.; Sawada, J.C.; Katoh, S.; Matsuno, K.; Ogo, N.; Ishikawa, Y.; Hashimoto, H.; Fujii, S.; Asai, A. Structural Basis of New Allosteric Inhibition in Kinesin Spindle Protein Eg5. ACS Chem. Biol. 2015, 10, 1128-1136. [CrossRef]

52. Kaan, H.Y.K.; Major, J.; Tkocz, K.; Kozielski, F.; Rosenfeld, S. Snapshots of Ispinesib-induced Conformational Changes in the Mitotic Kinesin Eg5. J. Biol. Chem. 2013, 288, 18588-18598. [CrossRef] [PubMed]

53. Rinaldi, J.; Wu, J.; Yang, J.; Ralston, C.Y.; Sankaran, B.; Moreno, S.; Taylor, S.S. Structure of Yeast Regulatory Subunit: A Glimpse into the Evolution of PKA Signaling. Structure 2010, 18, 1471-1482. [CrossRef]

54. Zhang, P.; Ma, J.; Zhang, Z.; Zha, M.; Xu, H.; Zhao, G.; Ding, J. Molecular basis of the inhibitor selectivity and insights into the feedback inhibition mechanism of citramalate synthase from Leptospira interrogans. Biochem. J. 2009, 421, 133-143. [CrossRef] [PubMed]

55. Thirumalai, D.; Lorimer, G.H.; Hyeon, C. Iterative annealing mechanism explains the functions of the GroEL and RNA chaperones. Prot. Sci. 2020, 29, 360-377. [CrossRef] [PubMed]

56. Ma, J.; Karplus, M. The allosteric mechanism of the chaperonin GroEL: A dynamic analysis. Proc. Natl. Acad. Sci. USA 1998, 95, 8502-8507. [CrossRef]

57. Hyeon, C.; Lorimer, G.; Thirumalai, D. Dynamics of allosteric transitions in GroEL. Proc. Natl. Acad. Sci. USA 2006, 103, 18939-18944. [CrossRef]

58. Yifrach, O.; Horovitz, A. Nested cooperativity in the ATPase activity of the oligomeric chaperonin GroEL. Biochemistry 1995, 34, 5303-5308. [CrossRef]

59. Horovitz, A.; Mondal, T. Discriminating between Concerted and Sequential Allosteric Mechanisms by Comparing Equilibrium and Kinetic Hill Coefficients. J. Phys. Chem. B 2021, 125, 70-73. [CrossRef]

60. Iwata, S.; Kamata, K.; Yoshida, S.; Minowa, T.; Ohta, T. T and R states in the crystals of bacterial L-lactate dehydrogenase reveal the mechanism for allosteric control. Nat. Struct. Mol. Biol. 1994, 1, 176-185. [CrossRef] [PubMed]

61. Rader, A.J.; Brown, S.M. Correlating allostery with rigidity. Mol. Biosyst. 2011, 7, 464-471. [CrossRef]

62. Blaszczyk, J.; Li, Y.; Yan, H.; Ji, X. Crystal structure of unligated guanylate kinase from yeast reveals GMP-induced conformational changes. J. Mol. Biol. 2001, 307, 247-257. [CrossRef]

63. Petit, C.M.; Zhang, J.; Sapienza, P.J.; Fuentes, E.J.; Lee, A.L. Hidden dynamic allostery in a PDZ domain. Proc. Natl. Acad. Sci. USA 2009, 106, 18249-18254. [CrossRef]

64. Kumawat, A.; Chakrabarty, S. Hidden electrostatic basis of dynamic allostery in a PDZ domain. Proc. Natl. Acad. Sci. USA 2017, 114, E5825-E5834. [CrossRef] [PubMed]

65. Kuo, L.C.; Zambidis, I.; Caron, C. Triggering of allostery in an enzyme by a point mutation: Ornithine transcarbamoylase. Science 1989, 245, 522-524. [CrossRef] [PubMed]

66. Kadirvelraj, R.; Sennett, N.C.; Polizzi, S.J.; Weitzel, S.; Wood, Z.A. Role of packing defects in the evolution of allostery and induced fit in human UDP-glucose dehydrogenase. Biochemistry 2011, 50, 5780-5789. [CrossRef] [PubMed] 